\title{
Calidad y cantidad en el análisis de las políticas públicas
}

\section{Geert Bouckaert*}

El concepto de "rendimiento" es un elemento clave en las estrategias de "modernización" del sector público de la OCDE y su evaluación. Estas modernizaciones aparecen en todos los países de la OCDE con diferentes formas, dependiendo de las culturas administrativas y políticas y usando diferentes etiquetas, como, por ejemplo, Nueva Gestión Pública (Hood, 1997), Gerencialismo (AucoIn, 1990; PoluIT, 1993), Neue Steurerung (KLAGES, 1998). El cambio también osćila entre lo sustancial, como, por ejemplo, Nueva Zelanda y lo incremental, como, por ejemplo, en Alemania (DERLIEN, 1998). Las preguntas clave son hasta qué punto están convergiendo o divergiendo las tendencias o trayectorias y qué tipo de ciclos de aprendizaje hay de estas experiencias de benchmarking (OtSON y PETERS, 1996).

Este cambio producirá impactos en los análisis de las políticas públicas, que pueden ser resumidos en tres proposiciones.

En primer lugar, se incrementará la cantidad de auditorías y evaluaciones de las políticas públicas.

En segundo lugar, puede que se incremente la calidad de las auditorías y de las evaluaciones.

En tercer lugar, habrá auditorías y evaluaciones de las cantidades y de las calidades.

La idea central de este trabajo es que la medición del rendimiento y de la gestión en el sector público es necesaria, útil y posible para las auditorías y evaluaciones en un contexto moderno de gobernación (governance).

La necesidad se relaciona predominantemente con las presiones económicas y con los vacíos sociales respecto de la confianza y la legitimidad. La utilidad debería detectarse en el nivel de integración de los ciclos financieros, por ejemplo, en la ca- dena de presupuestos, en los balances, en las auditorías y en los ciclos de la políticas públicas, como, por ejemplo, en la preparación, en la toma de decisiones, en la implementación y en la evaluación. La medición del rendimiento y la gestión es posible, pero existen todavía muchos problemas. Sin embargo, la medición del rendimiento (las técnicas) está evolucionando y progresando a un nivel macro (del país), medio (los ámbitos de las políticas públicas) y micro (la organización).

\section{La cantidad de auditorías y las evaluaciones}

Parece haber cuatro grandes tendencias en muchos países de la OCDE, aun cuando a algunos de ellos les gustaría tener una aproximación más incremental que sustancial, y a pesar de que hay países que tienen posturas más contenidas que radicales, por razones históricas o de otra índole.

Comúnmente se presupone que las Administraciones no cambian por sí mismas, sino que necesitan presión para que se produzca el cambio. Los llamados procesos de modernización consisten en organizar e institucionalizar estas presiones desde un punto de vista interno y externo. En ambos casos, se supone que estas presiones cambiarán la organización de las actividades y los valores que enjuician estas actividades, hasta el punto de mejorar el rendimiento de las organizaciones y de las políticas. In Search of Results ("En busca de Resultados") es una guía básica para las prácticas de gestión del rendimiento en muchos 
países de la OCDE y describe estas cuatro tendencias con detalle (BOUCKAERT, 1997; OCDE, 1997).

La primera tendencia es aumentar la presión externa integrando mecanismos de mercado (market-type-mechanisms o MTM) mediante el empleo (más sistemáticamente) de ofertas competitivas, cupones (vouchers), cuasi-mercados, mercados internos, etc. La postura más extrema es la de privatizar las actividades. Se supone que la presión externa aumentará el rendimiento de estas actividades. Probablemente, Nueva Zelanda y el Reino Unido fueron muy lejos en esta dimensión. El que estas evaluaciones periódicas de las políticas públicas y las auditorías de firmas privadas (subvencionadas) sean deseables es todavía un tema de discusión, ya que se supone que el mercado adoptará las "mejores prácticas" (OCDE, 1996).

La segunda tendencia es la de integrar técnicas de mercado en las organizaciones públicas. Esto se traduce en una creciente lista de métodos y técnicas aplicadas, como, por ejemplo, la fijación de los objetivos, la planificación estratégica y operativa, los contratos, el presupuesto del rendimiento, la "contabilidad acumulativa" (accrual accounting), las auditorías basadas en los modelos de value for money, etc. Estos métodos y técnicas ofrecen información relacionada con el rendimiento, lo que da como resultado una tensión entre la realidad y los estándares (otras unidades, objetivos, series temporales, etc.). Se supone que esta presión interna aumentará el rendimiento de estas organizaciones. El mecanismo para adoptar medidas más explícitas sobre la competencia de recursos y sobre las responsabilidades por los resultados, por ejemplo, en los contratos internos, requerirá mecanismos de responsabilización proporcional (proportional accountability), que requieren, de forma inmediata, auditorías y evaluaciones.

La tercera tendencia está relacionada con un cambio en las unidades, de unidades más centralizadas a unidades autónomas de menor tamaño, más descentralizadas o transferidas. Esto produce una proliferación y fragmentación de las actividades en las unidades organizativas parcialmente independientes, con a veces tareas homogéneas, que tienen sus propios presupuestos y contabilidades. Por lo tanto, esto también requiere una extensión de los mecanismos de auditoría para no perder el control sobre la miríada de organizaciones.

El cuarto cambio supone desemparejar los campos de las políticas públicas y los elementos claves de los ciclos de dichas políticas y ubicarlos en diferentes organizaciones. La etapa de implementación es una parte tradicional del ciclo de las políticas públicas, que (periódicamente) se atribuye a una organización diferente, dentro (en el presupuesto) o fuera (en el mercado) del gobierno. Segmentar el ciclo de políticas públicas en diferentes partes, según sus componentes (la preparación, la toma de decisiones, la implementación y la evolución) requiere, por razones de especialización, un esfuerzo adicional para coordinar estas partes en un ciclo. La auditoría y la evaluación serán esenciales para garantizar esto.

Todas las tendencias se inclinan a apoyar la proposición de que las auditorías lleguen a ser más funcionales y quizás, incluso indispensables para garantizar los "resultados" o el "rendimiento" y para detectar y controlar "el éxito y el fracaso". Como consecuencia, las auditorías internas son más visibles en algunas organizaciones y los Tribunales de Cuentas (Supreme Audit Institutions) están adaptando sus prácticas. Muchas instituciones auditoras, internas y externas, se están también involucrando cada vez más en las evaluaciones. El creciente éxito de la Sociedad Europea de Evaluación y su revista Evaluation es un claro indicador de este fenómeno.

\section{La calidad de las auditorías y de las evaluaciones}

Para poder auditar y evaluar es necesario contar con datos e información. El diseño y los modi operandi de los sistemas de medición del rendimiento son, por tanto, cruciales en la construcción de un marco válido y fiable de auditoría y evaluación.

En esta parte pondré énfasis en la importancia de contar con visiones convergentes sobre la medición del rendimiento a nivel macro, meso y micro, para llevar a cabo una evaluación sostenible. Se adoptará un enfoque centrado en las evoluciones de los sistemas de medición del rendimiento en estos tres niveles. Hasta el punto de que cuanto más convergentes y coherentes sean las evoluciones en estos niveles, más fuertes serán las iniciativas de auditoría y evaluación.

\subsection{Evoluciones en el nivel macro}

El nivel macro es el nivel de la sociedad donde el gobierno es un actor junto al sector privado, lucrativo y no-lucrativo. Esto se refiere, en último término, a una combinación, agregación y consolidación de los mercados, las jerarquías y los sistemas, donde se considera que el gobierno tiene una influencia potencial sobre la sociedad.

Un indicador importante de la economía nacional es el PIB. Existe un creciente debate sobre los déficit de tal indicador. En la medida en que el capital social y medioambiental y su funcionamiento no están incluidós, y que también se ignoran los 
compromisos futuros y los impactos, el PIB ofrece un paisaje incompleto de la macro evaluación.

En el nivel macro, existe un cambio desde la hipótesis cero a la hipótesis no-cero. La hipótesis cero significa que la participación del gobierno en el PIB se mide como un input. La operacionalización del valor del output es equivalente al valor de los recursos que producen este output. Dado que el output iguala al input, nunca puede haber un progreso ni un deterioro en la evaluación de la productividad del gobiemo en el nivel macro. Ésta ha sido la postura tradicional de un gobiemo consumista y era una postura cómoda para evitar todos los problemas de medición. Sin embargo, cuanto más produce un gobiemo activamente, mayor es su participación en la búsqueda de recursos para generar bienes y servicios y mayor es la necesidad que hay de determinar el valor añadido.

Desde el punto de vista del productor con respecto al gobierno, la hipótesis cero ya no es una solución estadística aceptable para estimar el output público y el rendimiento. El input produce el output, también en el sector público. Y el input no es igual al output, ni en términos físicos ni monetarios. La medición del rendimiento del gobierno y sus cambios debería mostrar cómo influyen los servicios y políticas gubernamentales en la visión de la panorámica nacional (The Swedish Ministry of Finance, 1987). También muestra la importancia de un sector público productor. El cambio de la hipótesis cero a la no-cero sobre la productividad del gobierno supone un cambio metodológico importante para la productividad en el nivel macro y para la evaluación de las intervenciones gubernamentales a nivel societario. Esto proporciona un peso inmediato a las estimaciones de rendimiento de los niveles micro e intermedio (meso) y a las evaluaciones de las agencias gubernamentales y, también, a los niveles que están consolidados y agregados en el nivel macro. Ejemplos de los esfuerzos para recalcular el nivel macro son, entre otros, los de EE.UU. (ILO), Suecia (MURRAY, 1997), Finlandia (LehtoranTa y Niemi, 1997) y los Países Bajos (SCP, 1997). La subsiguiente re-determinación o re-evaluación del nivel macro parece ser una parte más difícil.

Esta perspectiva macro se está desarrollando en países como, por ejemplo, Suecia (Proyecto-VESTA), Finlandia (proyecto de governance) y Canadá, donde los indicadores macro del rendimiento societario están en desarrollo, o en los Países Bajos, donde se ha trazado la función del gobierno de influenciar a la sociedad.

\subsection{Evaluaciones en el nivel intermedio (meso nivel)}

Ha habido tres momentos de cambios en el ámbito de la medición de los rendimientos en el nivel intermedio (meso nivel), que es el ámbito de las políticas públicas desde las organi- zaciones. Estas políticas públicas pueden traspasar, a menudo, las líneas fronterizas organizacionales.

Primeramente, se está produciendo una ampliación del alcance de la medición en diferentes campos de servicios y de políticas públicas. En segundo lugar, hay un cambio en el desarrollo del diseño y del contenido de los sistemas de medición, desde la disponibilidad de los datos a la deseabilidad de los mismos. Y en tercer lugar, la calidad se incluye y está vinculada de forma más sistemática a los datos cuantitativos.

En primer lugar, hay una ampliación en la gama de las prácticas de medición en varios campos de servicios y de políticas públicas. El uso de grupos de medidas de rendimiento rutinarias basadas en la producción, se exporta a todos los tipos de "producción" y se "imita" en todas las organizaciones. Asimismo, esta producción se consume y debería tener como resultado la satisfacrión del cliente. Una vinculación directa y positiva entre el input y el output y entre el output y el efecto o la satisfacción es un mecanismo implícito subyacente en el concepto de función de producción. Sin embargo, ¿cómo se relaciona esto con la "producción" del cuidado de los niños, o del cuidado de los ancianos, o del cuidado de los discapacitados mentales, o asimismo de la seguridad, de la investigación básica, o de las negociaciones de paz? Los servicios materiales, como, por ejemplo, la recogida de basura o la recaudación de impuestos, deberían considerarse como funciones de producción típicas. La producción atípica es un término que hace referencia al "procesamiento" de personas (o su comportamiento) o de ideas. Esos son servicios "relacionales" o de eideas". El objetivo de los servicios materiales es su producción y consumo tangibles y su correspondiente cadena de inputs, actividades, outputs y efectos. El objetivo de los servicios relacionales (como, por ejemplo, residencias para ancianos) es la asignación de roles. El objetivo de los servicios de ideas es su capacidad de convencer, como, por ejemplo, depósitos de ideas, investigación básica, negociaciones de paz). Hay un aumento de las prácticas de medición del rendimiento de lo tangible a lo relacional, y de lo relacional a lo ideal. (BOUCKAERT y HALACHMI, 1996).

En segundo lugar, en muchas organizaciones existe un déficit de medición. Las mediciones no encajan con las características de las actividades y/o no se protegen ciertas características. En estos casos, se crean medidas porque se dispone de datos. Sin embargo, el impulso hacia la gestión del rendimiento lleva a cambiar la disponibilidad de los datos como criterio para la selección de los sistemas de medición, a la deseabilidad de estas medidas, lo que la mayoría de las veces significa que se han de crear nuevas series temporales de datos. La deseabilidad significa que las medidas son útiles para la adopción y la gestión de políticas públicas. Los propósitos de la evaluación requieren que se produzcan ciertas clases de información y esto exige 
una reflexión y una metodología anticipadas (ex ante) (BoucKAERT, 1992).

En tercer lugar, la inclusión de la calidad en la medición está llegando a ser una práctica habitual. En los primeros estudios sobre la eficiencia, se omitía el aspecto de la calidad del output, no porque la gente implicada desconociera el concepto, sino porque no había una solución técnica inmediata de cómo medirlo.

La discusión se hace más difícil si se relacionan calidad y coste. Según BRADFORD y otros, "los gobiernos locales no han sido capaces de controlar los crecientes costes del input, especialmente mano de obra, recortando el uso de estos inputs por medio de ahorros significativos en las técnicas de producción. Las mejoras en la calidad del output ciertamente han tenido lugar, pero más bien han contribuido a estimular más que a reducir los niveles de gasto público (BRADFoRd et als. 1969: 188-189). Esto es una prueba de la hipótesis de la conocida como "enfermedad Baumol. Sin embargo, la proporción de la calidad sobre el incremento del coste no siempre se ha calculado en estos estudios. Se ofrecen diferentes soluciones en un contexto donde los precios no se conocen (PolutT y BouckaERT, 1995). En primer lugar, una solución es asumir que la calidad es igual y constante si se quiere comparar los servicios. En este caso, la calidad no debe medirse en absoluto, puesto que no proporcionará una respuesta diferenciadora. Una segunda posibilidad es no contabilizar el output por debajo de un cierto nivel de calidad. En tercer lugar, uno puede elegir organizaciones con similar calidad en los outputs. El cuarto enfoque consiste en medir la calidad explícitamente y relacionar esta información con la cantidad de output. El resultado sería un output analizado y sopesado en términos de su calidad. La quinta respuesta es medir de forma separada la calidad y presentarla como una dimensión independiente.

\subsection{Desarrollo en los análisis a nivel micro}

El nivel micro se refiere a la organización individual, a la agencia o a la administración y a sus subunidades. Establecer y evaluar el rendimiento en este nivel, a menudo, se lleva a cabo relacionándolo con otras entidades comparables. Las técnicas habituales son el benchmarking o las tarjetas de puntuación equilibrada (balanced score cards). Sin embargo, ni una técnica ni la otra solventan el problema de qué indicadores o medidas elegir. Una importante innovación se ha producido en la metodología de comparar recíprocamente observaciones. Una tendencia en la discusión sobre la productividad interpreta la función de producción no sólo como una relación de inputy output descrita por una función matemática, sino también como una frontera para una empresa o una agencia. Esta frontera repre- senta el máximo output posible para un input dado y el input mínimo posible para un output dado en un cierto nivel de tecnología. La función de producción se convierte en la mejor frontera práctica de todas las posibles relaciones input-output. Todas las observaciones están relacionadas con esta línea divisoria para determinar si estas relaciones son ineficientes y en qué medida. Mientras que la productividad se relaciona con la función de producción, la eficiencia está relacionada con esta frontera práctica óptima. La descripción de estas líneas divisorias puede ser paramétrica o no-paramétrica, determinista o casual. El desarrollo de las técnicas no-paramétricas de análisis de envolvimiento de datos (data envelopment analysis) o el free disposal bull comenzó a finales de los años setenta y principios de los ochenta. El DEA (data envelopment analysis) fue desarrollado por primera vez por CHARNES et al. En 1978. El FDH (free diponsal bull), basado también en la obra de FARREL (1957), fue desarrollado por Deprins, Sima y Tulkens (1984). Desde finales de los años ochenta y principios de los noventa las técnicas de DEA y de FDH se usan cada vez más en el sector público.

\subsection{Conclusión}

Los sistemas de medición deben estar presentes y en funcionamiento en todos los niveles, macro, meso y micro. Estos sistemas también deben ser compatibles, puesto que se refieren unos a otros y se construyen unos a partir de otros, a través de mecanismos de agregación, consolidación o comparación. Estas compatibilidades son cruciales para establecer un marco apropiado de evaluación.

\begin{tabular}{|c|c|c|c|}
\hline \multicolumn{4}{|c|}{ 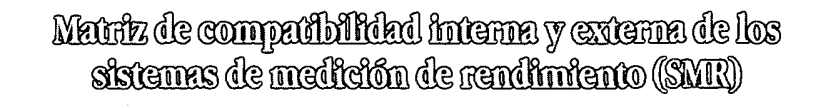 } \\
\hline $\begin{array}{l}\text { Compatibilidad } \\
\text { de SMR/Niveles } \\
\text { de Análisis }\end{array}$ & Organización & $\begin{array}{l}\text { Políticas } \\
\text { Públicas }\end{array}$ & Sistema \\
\hline SMR Micro & Auditorías & $\begin{array}{l}\text { Consolidación } \\
\text { interorganizativa }\end{array}$ & $\begin{array}{l}\text { Mercado } \\
\text { Redes jerárquicas } \\
\text { Agregación }\end{array}$ \\
\hline SMR Meso. & & Evaluaciones & $\begin{array}{l}\text { Consolidación } \\
\text { entre políticas }\end{array}$ \\
\hline SMR Macro & & & $\begin{array}{l}\text { Evaluación de la } \\
\text { noumernance }\end{array}$ \\
\hline
\end{tabular}

Debe haber un nivel de compatibilidad interna y externa de los sistemas de medición. Hay tres criterios para estas compatibilidades. Los sistemas de medición del rendimiento, en un contexto de evaluación, deben ser técnicamente válidos y fiables (y contar con los otros rasgos técnicos de las medidas y de la medición). También deben ser funcionales, lo que significa que la medi- 
ción debe ir encaminada a los objetivos de las organizaciones, de las políticas y de los sistemas. Tanto es así que si hay un prejuicio o una desviación potencial, la medición puede llegar a ser disfuncional. En tercer lugar, la medición debe ser legítima, lo que significa que debe ser financiada y apoyada por aquellos a los que se aplica. Éstos pueden ser el público en general, los consumidores, o incluso la propia generación.

De estos criterios se puede deducir, de forma inmediata, que la medición a todos los niveles, como ocurre con la evaluación, no es solamente técnica, sino que posee una carga valorativa y, por tanto, política.

Es importante aspirar a esta situación ideal y tratar de fusionar el nivel micro, meso y macro en el contexto de la evaluación.

Un equipo completo de herramientas de evaluación requiere de una visión coherente y convergente y practicar la medición del rendimiento en el nivel micro, meso y macro. La práctica de la evaluación también será la parte tan fuerte, como débil de la cadena. La política y el ciclo financiero podrían ser una buena operacionalización para valorar la fuerza de esta cadena.

La evaluación ex ante, ex nunc y ex post se refiere a los ciclos financieros y de la política. La evaluación ex ante alude a la preparación de la política y los presupuestos que a veces dan lugar a contratos. La evaluación ex nunc se refiere a las continuas prácticas de implementación, donde los sistemas de responsabilidad (y la información existente en el almacén de datos del sistema de responsabilidad por los costes) suministran la información indispensable. La evaluación ex post se refiere a las auditorías. Todo esto puede analizarse desde una perspectiva micro, meso y, finalmente, macro.

En la medida en que los sistemas de medición del rendimiento al nivel micro, meso o macro no encajan entre sí de forma complementaria, no serán posibles las agregaciones y consolidaciones o comparaciones entre la información al mismo nivel de análisis (por ejemplo, todos los municipios) y entre todos los diferentes niveles de análisis (por ejemplo, las regiones y el país; o las políticas y las cuotas del gobierno en el PIB).

Las soluciones pragmáticas de la descentralización y las agencias que están apoyadas por la ideología de la Nueva Gestión Pública obligan a centrarnos en el nivel de la unidad organizativa, que es el nivel micro. Al desarrollar los contratos y separar los sistemas de responsabilidad, uno obtiene una información muy detallada sobre las unidades más pequeñas. Esto crea la ilusión de tener el control de las partes y, por tanto, de toda la maquinaria del gobierno. El gobierno por contratos ofrece una visión fragmentada, en la cual una miríada de contratos permite una visión microscópica clara. La suposición es que si uno detenta el control de estas partes, automáticamente puede consolidar y asumir que controla la totalidad del sistema.
Sin embargo, la cuestión estriba en hasta qué punto hay una desconexión entre el nivel micro y el meso, y entre el meso y el macro. El indicador más importante es hasta qué punto hay una desconexión entre las políticas y los planes de gestión y entre los outputs y los resultados.

La Nueva Gestión Pública puede producir mejoras en el nivel micro y oculatr deterioros y debilidades estructurales en el sistema global. No es una coincidencia que la gobernación (governance) se desarrolle paralelamente a la Nueva Gestión Pública.

La solución de Nueva Zelanda fue desarrollar las Áreas de Resultados Clave y las Áreas de Resultados Estratégicos. Los componentes más importantes de un Sistema de Medición de la Gestión llegaron a ser los alfileres entre el nivel micro, meso y macro en el contexto de la evaluación. Varios países han hecho algunos esfuerzos por consolidar la información en un nivel superior de análisis (planes de negocio consolidados, presupuestos consolidados, megadepartamentos).

\section{La auditoría y la evaluación de la calidad y la cantidad}

La calidad y cantidad de la auditoría tiene dos aspectos principales: la auditoría de la calidad y cantidad de información y la auditoría del contexto, los resultados obtenidos. Aquí el énfasis está en una primera parte técnica de una necesidad cada vez mayor de auditar la gestión, relacionada con la información.

El segundo elemento engloba la evaluación del programa, que supone ir más allá o detrás de las medidas de gestión organizacional. "Abarca una valoración en profundidad de los costes y beneficios del programa, no todos los cuales pueden cubrirse con las medidas de gestión o indicadores. También puede suponer revisar la adecuación de los objetivos del programa. Intentará evaluar y analizar las vinculaciones entre los diferentes inputs, procesos, outputs y resultados para comprender la lógica del nivel concreto de gestión. Mientras que en este sentido pueden examinarse todas las dimensiones de la gestión (economía, eficiencia, efectividad, calidad de servicio, gestión financiera), la práctica se concentra en el nivel superior de la efectividad del programa" (BOUCKAERT en la OCDE, 1994, p. 41).

Un número cada vez mayor de oficinas de auditoría interna y externa también se centran en la calidad de los datos. En Finlandia, la Oficina de Auditoría del Estado se centra en la exactitud y adecuación de la información externa de las agencias en 
lo que respecta a los resultados". Esta información se usa en los contratos e informes anuales que están sujetos a revisión. En Suecia, las auditorías de la Oficina de Auditoría Nacional aluden a las fuentes de datos, el procesamiento, la adecuación de los métodos empleados, la relevancia de la información y las conclusiones. Sin embargo, ésta no es una práctica común y está sujeta a desarrollo.

\section{4. ¿Hacen "mejor" a las políticas públicas más y mejores auditorías y evaluaciones?}

Hay varias razones por las cuales los sistemas de medición de la gestión no son óptimos y por las que no hay una integración óptima de las auditorías y la evaluación en los ciclos de las políticas públicas. Las razones pueden variar desde el nivel psicológico, técnico, político e institucional, hasta los rasgos culturales de los sistemas.

La razón psicológica es compleja. El estudio de la codificación y recuperación de los datos numéricos para la toma de decisiones en los contextos de gestión es raro. KIDA y SMITH (1995, p. 585) señalan que ulas reacciones afectivas o evaluativas ante los datos numéricos son centrales para los procesos de codificación y recuperación en los contextos de responsabilidad por las decisiones. Demuestran que "cuando los que toman las decisiones observan los datos numéricos, típicamente establecen comparaciones entre los datos y determinan reacciones evaluativas. De acuerdo con esto, los datos numéricos son inicialmente codificados de formas diferentes. Y lo que es más, dentro de la forma de reacción afectiva, los individuos determinan y codifican reacciones afectivas a las construcciones o amplias categorías de datos, alli donde es posible. Mientras que los datos pueden ser codificados de formas distintas, los recuerdos resultantes no se consideran igualmente recuperables" (p. 602). Su modelo demuestra que el afecto evaluativo es la forma más recuperable de los recuerdos, como el tipo más accesible de reacción afectiva y tiene una gran confianza en recordar el afecto evaluativo.
La aproximación de la Administración pública a la toma de decisiones ofrece una explicación complementaria (OTTEN, 1996). Resulta evidente que la suposición de suministrar información correcta sobre la gestión en los contextos evaluativos es necesaria, pero no suficiente para evitar una toma de decisiones catastrófica. Desconocer la información o una lectura selectiva y discriminatoria es el comportamiento común de los que toman las decisiones. Los complejos proyectos técnicos (sistemas de armas, plantas de energía nuclear, ICT) o las políticas (guerras sobre la pobreza) a menudo continúan existiendo y se desarrollan posteriormente siguiendo el mismo curso, incluso si todos los datos existentes, la información y los informes sugieren un cambio necesario en la política.

Los patrones culturales también ayudan a explicar la carencia de integración (HoOD, 1996). Los sistemas de reciprocidad se referirán a evaluaciones semejantes. Los sistemas basados en la competición no necesitarán inexorablemente evaluaciones, ya que, según algunos observadores, el mercado y la competición se supone que garantizarán "la mejor práctica". Los sistemas basados en la revisión tendrán mecanismos y herramientas de auditoría muy desarrollados. Finalmente, los sistemas basados en la casualidad imaginada prestarán atención a los procesos internos y pensarán en términos de medias que a largo plazo deberán dar una respuesta adecuada y tenderán a una buena evaluación media. El mecanismo de coordinación de la "jerarquía a través de la revisión" está siendo reemplazado cada vez más por aquellos relativos a la ueficiencia a través de la competición" y el "consenso a través de la reciprocidad" con la potencialidad de algunos conflictos.

La auditoría y la evaluación tienen por objetivo la mejora que deberá detectarse a través de los sistemas de medición de la gestión. Una estrategia importante para mejorar la práctica de la auditoría, la evaluación y la práctica de medir la gestión es centrarse en los círculos de aprendizaje a todos los niveles, micro, meso y macro. La medición, la auditoría y la evaluación están interrelacionadas para mejorar a través de la construcción y la práctica de aprender del sistema.

La medición y la evaluación detectan ciertas posiciones indeseables y la información ofrecida puede iniciar, aumentar o dirigir un proceso de cambio y adaptación hacia una posición mejor. Este cambio puede seguir un camino de aprendizaje de primer orden o de segundo orden. 
- Profesor de Gestión Pública y director del Centro de Gestión Pública en la Universidad Católica de Lovaina, Bélgica. Su campo de especialización es el de los sistemas de medida del rendimiento y los ciclos de gestión financiera en el sector público (email: geen.Bouckaert@soc.kueleven.ac.be).

\section{Bibliografia}

Aucon, P. (1990), Administrative Reforms in Public Management. Governance 3(2), pp. 115-137.

BoucKaerT, G. y HalachM, A. (1996), The Range of Performance Indicators in the Public Sector: Theory vs Practice. En HaLACHMI, A. y GRANT, D. (Eds.), Reengineering and Performance Measurement in Criminal Justice and Social Programmes. Perth, Western Australia, Ministry of Justice, pp. 91-106.

BoucKaerT, G. (1992), ‘Public Productivity in Retrospective. En Holzer, M. (Ed.), Public Productivity Handbook. New York, Marcel Dekker, pp. 15-46.

(1994), ‘Performance Measurement. En OECD, Performance Management: Performance Measurement and Results Oriented Management. Paris, PUMA, OECD.

_(1997), Overview and Synthesis. En OECD, In Search of Results: Performance Management Practices. Paris, PUMA, OECD.

(1997), Sustainable development of Networks in a Governance Context. Paper delivered at the conference on Spanning the Glober Divide: Networking for Sustainable Delivery. Steellenbosch University, South Africa, September.

(1998), .Public Sector Performance Management in a Principal-Agent Context: Some Reflections. En HALACHMI, A. y BORSMA, P. (Eds.), Inter and Intra Gobernment Arrangements for Productivity. Dordrecht, Kluwer Academic Publichser, pp. 137-145.

CHARNES, A.; COOPER, W. y THODES, E. (1978), Measuring Efficiency of Decision Making Units. European Journal of Operations Research, 2, pp. 429-444.

Deprins, D.; Simar, L. y Turkens, H., "Measuring Labor-efficiency in Post Offices. En Marchand, M.; Pestiau y Tulkens, H. (eds.), The Performance of Public Enterprises: Concepts and Measurements. Elsevier: North-Holland, pp. 243-267.
DERLIEN, H.-U. (1998), From Administrative Reform to Administrative Modernization, Verwaltungswissenscaftliche Beiträgue 33, Universität Bamberg.

Hood, Chr. (1997), Remedies for Misgovemment: Changing the Mix, but not the Ingredientes?. Keynote Speech at the European Group of Public Administration Conference, Leuven, 1997.

KLAGES, H. (1998), •Verwaltungsmodemisierung, "Harte" und "Wiche" Aspekte II. Speyerer Forshungberichte 181, Speyer.

Lehtoranta, O. y Niemi, M. (1997), •Measuring Public Sector Productivity in Finland: Progress Report. OECD-UNECE-EUROSTAT Meeting of National Accounts Experts, Paris, June (STD/NA(O7)15.

Murray, R. (1997), .Public Sector Productivity in Sweden. Papers on Public Sector Budgeting and Management in Sweden, Vol. 3. Stockholm, Budget Department, Swedish Ministry of Finance.

NIEMI, M. (1998), •Measuring Government Sector Productivity: Productivity Change between years 1995 and 1996 in Central Government. Internal Document of Statistics Finland.

OECD (1994), Performance Management: Performance Measurement and Results Oriented Management. Paris, PUMA, OECD.

OLSON, J. y PETERS, G. (Eds.) (1996), Lesson from Experience. Experiential Learming in Administrative Reforms in Eight Democracies. Oslo, Scandinavian University Press.

PounT, Chr. (1993), Managerialism and the Public Sector. Blackwell, London. 


\section{Cuadernos de}

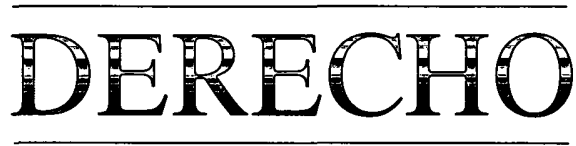

\section{PUBLICO}

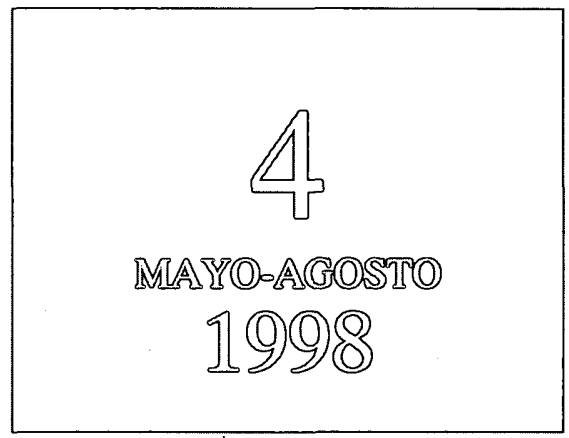

\section{INAP}

\section{Instituto Nacional de Administración Pública}

Nueva publicación editada por el Instituto Nacional de Administración Pública, los Cuadernos de Derecho Público, dedicada a los estudios y análisis relativos a la organización y funcionamiento del Estado desde una perspectiva global, interdisciplinaria, que aspira como tal publicación, integradora y rigurosa en sus planteamièntos, a abrirse un espacio en el marco de la publicística actual.

\section{CONSEJO DE REDACCIÓN}

Presidente: José Luis Meilán Gil. Vocales: Enrique Arnaldo Alcubilla, José Luis Cascajo Castro, Francesc de Carreras Serra, Ignacio Díez Picazo, Germán Fernández Montalvo, Tomás González Cueto, Lourdes Máiz Carro, Alejandro Menéndez Moreno, Juan Antonio Ortega Díaz-Ambrona, José Luis Piñar Mañas, María Rosa Ripollés Serrano, Luis Ignacio Sánchez Rodríguez, José Antonio Souto, Carles Viver i Pi-Sunyer, Tomás S. Vives Antón.

\section{Director}

Luis Aguiar de Luque

\section{Secretario}

Ricardo Blanco Canales

\section{Coordinación}

Centro de Publicaciones del INAP

Edita:

MINISTERIO DE ADMINISTRACIONES PÚBLICAS

Instituto Nacional de Administración Pública

ISSN: $1138-2848$

NIPO: $329-97-007-1$

Depósito Legal: M-35.142-1997

Imprime: Solana e Hijos, A.G., S.A.

San Alfonso, 26. 28917 Madrid

\section{Precios:}

Suscripciones y Administración:

Instituto Nacional de Administración Pública

Publicaciones Periódicas

Atocha, 106. 28012 Madrid

Teléfono: 3493235 